\title{
INFRAESTRUCTURAS DE DATOS ESPACIALES: DESARROLLO DE HABILIDADES ESPACIALES EN EL ENTORNO DEL ESPACIO EUROPEO DE EDUCACIÓN SUPERIOR'
}

\author{
Carlos Carbonell Carrera \\ Departamento de Expresión Gráfica en Arquitectura e Ingeniería. Universidad de La Laguna \\ Miguel Ángel Mejías Vera \\ Departamento de Geografía. Universidad de La Laguna \\ José Luis Saorín \\ Departamento de Expresión Gráfica en Arquitectura e Ingeniería. Universidad de La Laguna \\ Manuel Contero González \\ Departamento de Ingeniería Gráfica. Universidad Politécnica de Valencia
}

\section{RESUMEN}

En este trabajo se muestran los resultados de un estudio piloto destinado a estudiantes universitarios en el que se analiza la interacción de las Tecnologías de Información Geográfica con el desarrollo de las habilidades espaciales. Usando el Geoportal de la Infraestructura de Datos Espaciales de España se realizan una serie de ejercicios al objeto de estudiar su influencia sobre las capacidades espaciales, para lo cual se emplean los test DAT-SR, MRT y Perspective Taking/Spatial Orientation Test.

Palabras clave: Habilidades espaciales, Infraestructura de Datos Espaciales, Espacio Europeo de Educación Superior.

Fecha de recepción: julio 2010.

Fecha de aceptación: febrero 2012.

1 Trabajo realizado dentro del Proyecto de Investigación «Evaluación y Desarrollo de Competencias Asociadas a la Capacidad de Visión Espacial en los Nuevos Títulos de Grado en Ingeniería», Ref.: EA2009-0025, financiado por el Ministerio de Educación y Ciencia. Investigador Principal: Dr. José Luis Saorín Pérez. 


\section{ABSTRACT}

In this work the results from a pilot study focused on university students are shown analyzing interaction between Geographic Information Technologies and development of spatial abilities. About the geoportal of Spain's Spatial Data Infrastructure, several exercises are completed for studying its influence over spatial skills where DAT-SR, MRT and Perspective Taking/Spatial Orientation tests are used.

Key words: Spatial Skills, Spatial Data Infrastructure, the European Higher Education Area.

\section{INTRODUCCIÓN}

La aparición de las Tecnologías de la Información y Comunicación (TIC) en el ámbito geográfico y cartográfico supone un cambio de formato del profesional en su entorno de trabajo. Estas tecnologías han tenido un gran impacto tanto en la Geografía como ciencia como en la Educación Geográfica (Durán, 2005; Herrero, 2001; Rodríguez, 2003; Villanueva, 2003).

Nuevos interfaces, nuevos soportes digitales de información territorial constituyen un escenario en el que geógrafos, ingenieros y demás colectivos relacionados con la cartografía y ciencias afines comparten información georeferenciada a través de los Sistemas de Información Geográfica. (Torres, 2003).

En el ámbito universitario, los objetivos de los nuevos grados en Geografía e Ingeniería del Espacio Europeo de Educación Superior contemplan el desarrollo de habilidades específicas relacionadas con la obtención, análisis, tratamiento y representación de información geográfica y cartográfica (R.D. 55/2005 de 21 de enero y R.D. 1393/2007 de 30 de octubre).

No se disponen datos, en cambio, que acrediten si existe una correlación entre el desarrollo de estas habilidades específicas con el empleo de nuevos interfaces de información geográfica.

En este artículo vamos a analizar las componentes de la habilidad espacial y su desarrollo con el uso de nuevas Tecnologías de Información Geográfica en colectivos en los que las habilidades de visión espacial suponen un factor determinante en sus futuras posibilidades de éxito en el campo profesional (McGee, 1979; Miller, 1996; Smith, 1964; Martín Dorta, et. al., 2008), como son ingenieros y geógrafos.

A tal efecto se diseña un curso de corta duración en el que estudiantes de Ingeniería y Geografía manejan un interface de información geográfica sobre el que se les propone una serie de ejercicios y se les somete a una batería de test que miden sus habilidades espaciales.

Previamente se realiza una recogida de datos sobre el uso y conocimiento de las nuevas tecnologías de información geográfica por parte del alumno y, al final del ensayo, se realiza una encuesta de satisfacción sobre el curso realizado. 


\section{Nuevas tecnologías de información geográfica: las infraestructuras de datos espaciales}

En el marco de difusión de Información Geográfica, el concurso de las nuevas Tecnologías de la Información y Comunicación (TIC) ha dado lugar a diversos soportes de información geográfica: Sistemas de Información Geográfica (SIG), Infraestructuras de Datos Espaciales (IDE), Virtual Globes como Google Earth, Google Maps... acuñando el término Tecnologías de la Información Geográfica (TIG), definidas como todas aquellas disciplinas que permiten generar, procesar o representar información geográfica (Mejía, 2008).

La Tecnología de Información Geográfica empleada en este estudio es la Infraestructura de Datos Espaciales de España (IDEE). Este servicio integra datos, metadatos, servicios e información de tipo geográfico producido en España. A través de Internet, con acceso libre y gratuito, en el Geoportal de la IDEE (<http://www.idee.es $>)$ se integran los nodos y geoportales de recursos IDE de productores de información geográfica a nivel nacional, regional y local, así como una gran variedad de datos y servicios de información geográfica.

Este servicio, impulsado por la Agencia Europea de Medio Ambiente y Eurostat en consonancia con la iniciativa europea INSPIRE (Infraestructure for Spatial Information in Europe, Directiva 2007/2/CE) para la formación de una Infraestructura Europea de Datos Espaciales, fue aprobado por la Comisión Permanente del Consejo Superior Geográfico el 10 de abril de 2002. A partir de entonces se coordinó, por parte de la Comisión de Geomática, el desarrollo de una Infraestructura Nacional de Datos Espaciales que integrara todas las Infraestructuras de Datos Espaciales desarrolladas por productores oficiales de datos a nivel nacional, regional y local, así como de todo tipo de infraestructuras sectoriales y privadas.

Al realizarse el estudio en la Universidad de La laguna, en la isla de Tenerife, se eligió, dentro del Geoportal de la IDEE, la Infraestructura de Datos Espaciales de Canarias IDE Canarias.

\section{La Habilidades Espaciales y su medición}

La capacidad espacial es aceptada por distintos autores a través de la historia como una componente de la inteligencia. Es preciso, no obstante, distinguir entre aptitud, habilidad y capacidad espacial.

La Aptitud Espacial se define como el potencial innato que un individuo tiene para visualizar, previo a que pueda realizar cualquier tipo de entrenamiento que pueda afectarle. En cambio, la Habilidad Espacial puede ser adquirida a través del entrenamiento. Algunas personas disponen de un mayor grado de aptitud innata, pero la gran mayoría puede adiestrar esta habilidad a través de la práctica (Sorby et al., 2003).

La Capacidad Espacial integra aptitudes habilidades y/o destrezas (Sánchez y Reyes, 2003) y se entiende como la habilidad de manipular mentalmente los objetos y sus partes en un espacio bidimensional y tridimensional (Martín Dorta, 2009).

En este artículo se emplea el término de habilidad espacial para referirnos a la parte de la capacidad espacial que podemos adiestrar mediante entrenamiento.

A la hora de estudiar los componentes de la habilidad espacial encontramos en nuestras consultas bibliográficas distintos enfoques, entre los que no hay consenso, para establecer su 
clasificación y, a su vez, varias pruebas para obtener resultados cuantitativos a través de test dedicados a medir la visión espacial.

Una de las clasificaciones más recientes es la que establece Rovilson Mafalda (2000), quien propone tres componentes de la habilidad espacial:

- Relaciones espaciales: habilidad para evaluar si dos imágenes rotadas de manera diferente representan un mismo objeto (Mafalda, 2000), o bien, habilidad de rotar en nuestra imaginación, rápida y acertadamente, figuras de dos o tres dimensiones (Linn y Petersen, 1985), autores que se decantan por el término de Rotación espacial en lugar de Relación espacial.

- Orientación espacial: habilidad para evaluar cómo una secuencia de movimientos espaciales pueden ser representados desde diferentes orientaciones (Mafalda, 2000). Otros autores apuntan definiciones de este factor en torno a la capacidad de imaginar el aspecto de objetos desde diferentes orientaciones del observador (McGee, 1979), o bien, la habilidad para orientarse en relación al entorno y la conciencia de ubicación de uno mismo (Reber, 1985).

- Visualización espacial: este factor mide un proceso de aprendizaje, encadenamiento y manipulación mental de formas espaciales (Mafalda, 2000). Se define también como la habilidad de manipular información visual compleja cuando para producir una solución correcta se necesitan varias etapas (Linn y Petersen, 1985), asociado al término visión espacial.

Maier (1998), en cambio, ofrece cinco componentes principales de las habilidades espaciales:

- Relaciones espaciales - Spatial Rotations (SR): se refiere a la percepción de la posición de un objeto en relación a una posición anterior, considerando tamaño, distancias, volumen o cualquier otro signo distintivo.

- Percepción espacial - Spatial Perceptions (SP): es la capacidad para determinar las relaciones espaciales entre objetos, a pesar de la existencia de información que no es significativa.

- Visualización espacial - Spatial Visualization (SV): es la capacidad de manipular mentalmente las imágenes visuales. Esto puede implicar imaginar las rotaciones de objetos en el espacio.

- Rotación mental - Mental Rotation (MR): se refiere a la capacidad de rotar mentalmente imágenes visuales. Estas imágenes pueden ser bidimensionales o tridimensionales.

- Orientación espacial - Spatial Orientaion (SO): es la capacidad de orientarse física o mentalmente en el espacio. La posición espacial de una persona es esencial para esta tarea.

Estudios llevados a cabo con alumnos de Ingeniería en la Universidad de La Laguna se centraban en la medición de dos componentes: relaciones espaciales y visión espacial, adoptando una clasificación simplificada en dos categorías (McGee, 1979; Burnet \& Lane, 1980; Pellegrino et al., 1984; Clements \& Batista, 1992; Olkun, 2003):

- Relaciones espaciales: habilidad de realizar rotaciones y comparaciones en cubos bidimensionales y tridimensionales (incluyendo las rotaciones espaciales y la percepción espacial de la clasificación de Linn \& Petersen). 
- Visión espacial: habilidad de reconocer piezas tridimensionales mediante plegado y desplegado de sus caras.

Estos trabajos se basaban en diferentes estrategias y herramientas en las que el estudiante interactuaba con modelado de piezas en tres dimensiones: modelado por ordenador basado en bocetos (Saorín, 2006) modelado 3D con software específico (Google SketchUp) y dispositivos de pantalla táctil (Martín-Dorta, 2009), Videoconsolas, Videojuegos y Realidad Aumentada (Martín, 2010). En estos estudios no se había considerado la orientación espacial.

Con la incorporación de la cartografía y la información territorial en un entorno virtual como una herramienta más en el ejercicio de la profesión de Ingeniero, y considerando otros colectivos profesionales que hacen uso ella como son los Geógrafos, el estudio de una tercera componente como la Orientación Espacial entendemos que es necesario.

$\mathrm{Al}$ analizar en nuestro ensayo el desarrollo de las habilidades espaciales dentro de un entorno geográfico y cartográfico, es la clasificación de Mafalda (2000) la que se ha considerado en la parte experimental de este trabajo para medir las habilidades espaciales, al contemplar la orientación espacial como una de las componentes de la habilidad espacial junto con las otras dos componentes (Relación espacial y Visualización espacial).

En torno a la clasificación elegida, y al objeto de medir cada una de las componentes, hemos considerado tres tipos de test (Tabla 1):

- Mental Rotation Test (MRT) (Vanderberg \& Kuse, 1978), para medir la componente de Rotación Espacial. Empleado en experiencias previas (Devon et al., 1994; Sorby \& Baartmans, 2000; Gerson et al., 2001; Saorín, 2006, Martín-Dorta, 2009; Martín, 2010).

Tabla 1

COMPONENTES DE LA CAPACIDAD ESPACIAL Y TEST DE MEDIDA

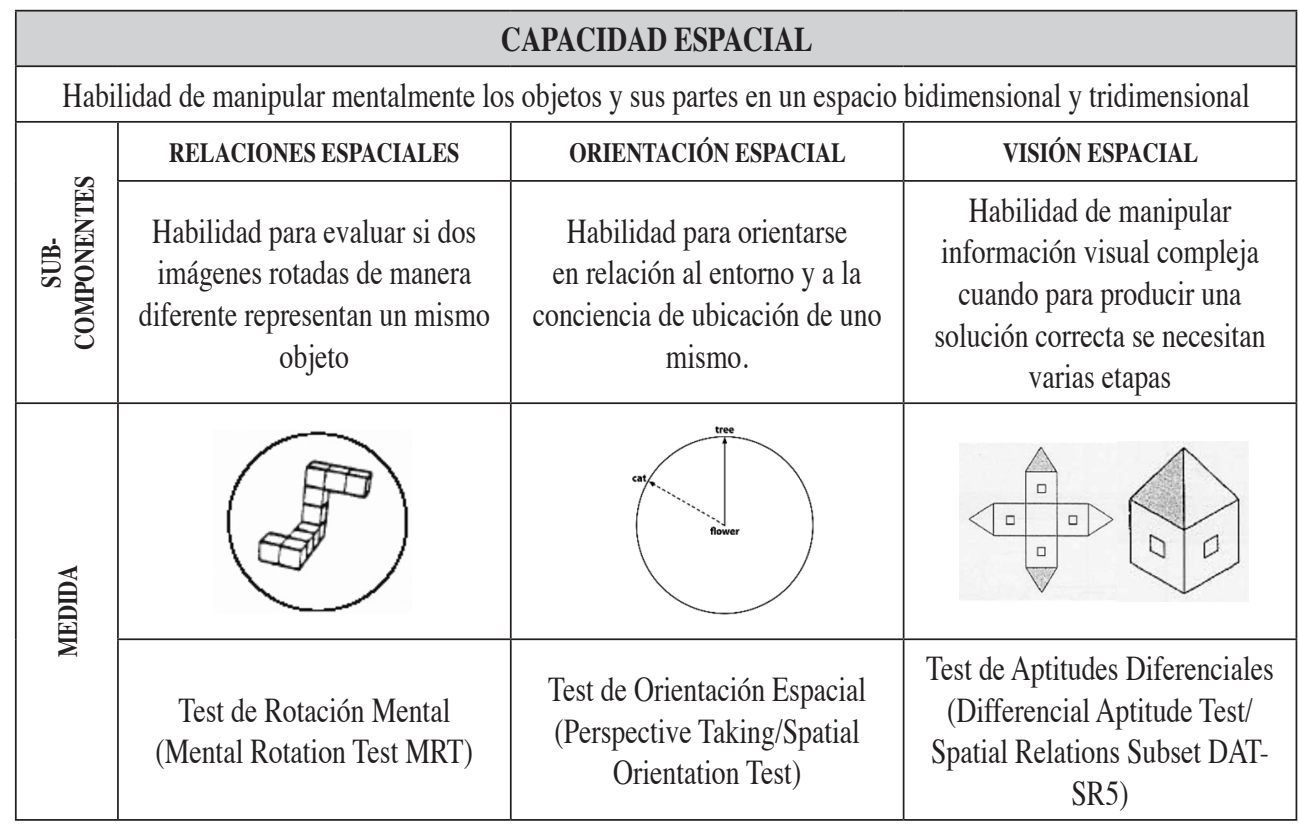


- Perspective Taking / Spatial Orientation Test (Hegarty et al., 2008), para medir la componente de Orientación espacial. Empleado en experiencias previas (Hegarty \& Waller, 2003, Kozhevnikov \& Hegarty, 2001, Hergarty, et al., 2008)

- Differencial Aptitude Test - Spatial Relations Subset (DAT-SR5) (Bennet, et al., 2000), para medir la componente de visión espacial. Empleado en experiencias previas (Devon et al., 1994; Sorby \& Baartmans, 2000; Gerson et al., 2001; Saorín, 2006, Martín-Dorta, 2009; Martín, 2010).

\section{OBJETIVOS}

Este estudio piloto se diseña al objeto de comprobar si el concurso de nuevas Tecnologías de la Información Geográfica desarrollan las habilidades espaciales en colectivos en los que, para el ejercicio de la profesión, es preciso disfrutar de un buen nivel de dichas habilidades (Ferguson, 1992). Se realizará, a su vez, un análisis de satisfacción del usuario en relación a la metodología y contenidos desarrollados.

\section{Hipótesis de trabajo}

Las hipótesis de trabajo de las que partimos son las siguientes:

- Un curso de corta duración utilizando Infraestructuras de Datos Espaciales es una herramienta válida para el objetivo de mejorar las habilidades espaciales, y en concreto de la Orientación Espacial.

- El empleo de nuevas herramientas tecnológicas de aprendizaje mejora la motivación del alumno.

Para poder validar las hipótesis de trabajo se han diseñado una serie de ejercicios prácticos a realizar con los alumnos. Se fijará una hipótesis nula $(\mathrm{H} 0)$ y se validará o no la suposición a través de métodos de inferencia estadística. Se aportan, a su vez, datos de encuestas de satisfacción que ayudan a confirmar la segunda hipótesis.

\section{METODOLOGÍA}

\section{Recogida de datos previa al curso}

Antes de comenzar la prueba piloto se realiza una recogida de datos sobre los alumnos participantes en el curso donde se obtiene el grado de penetración de las Tecnologías de Información Geográfica. Se le consultó al alumno sobre la frecuencia y el uso que hacía de Google Earth, IDE Canarias y, del formato tradicional de información cartográfica, el mapa en soporte de papel, al objeto de establecer una comparación de las nuevas tecnologías con los formatos tradicionales. (Tabla 2)

Podemos destacar el hecho de que la frecuencia de uso de las Infraestructuras de Datos Espaciales por el alumnado encuestado es muy pequeña, un $75 \%$ no lo ha usado nunca, mientras que el uso de Google Earth y Cartografía en formato papel está más generalizada. La búsqueda de lugares es la aplicación más solicitada indistintamente en cualquiera de los tres formatos. 
Tabla 2

FRECUENCIA Y USO DE CARTOGRAFÍA EN FORMATO PAPEL, GOOGLE EARTHE IDE CANARIAS

\begin{tabular}{|c|c|c|c|c|c|c|c|}
\hline Frecuencia & Cartografía & $\begin{array}{c}\text { Google } \\
\text { Earth }\end{array}$ & $\begin{array}{c}\text { IDE } \\
\text { Canarias }\end{array}$ & Uso & Cartografía & $\begin{array}{c}\text { Google } \\
\text { Earth }\end{array}$ & $\begin{array}{c}\text { IDE } \\
\text { Canarias }\end{array}$ \\
\hline Todos los días & $5 \%$ & $0 \%$ & $2 \%$ & Buscar lugares & $72 \%$ & $64 \%$ & $52 \%$ \\
\hline Alguna vez/semana & $11 \%$ & $33 \%$ & $5 \%$ & Medir dist./sup. & $11 \%$ & $10 \%$ & $19 \%$ \\
\hline Alguna vez/mes & $33 \%$ & $42 \%$ & $9 \%$ & Vistas 3D & $0 \%$ & $21 \%$ & $0 \%$ \\
\hline Alguna vez/año & $26 \%$ & $16 \%$ & $9 \%$ & Trabajo & $11 \%$ & $3 \%$ & $5 \%$ \\
\hline Nunca & $25 \%$ & $9 \%$ & $75 \%$ & Otros & $6 \%$ & $2 \%$ & $24 \%$ \\
\hline
\end{tabular}

\section{Prueba Piloto}

El curso se diseña atendiendo experiencias previas en cursos similares en la Universidad de La Laguna (Contero, et.al., 2005; Contero, et.al., 2006, Martín-Dorta 2009, Martín 2010), centrados en torno al desarrollo de habilidades de relaciones espaciales y de visualización espacial. Al analizar, en este caso, el impacto de las nuevas tecnologías de información geográfica e introducir un formato cartográfico era preciso considerar una componente de la habilidad espacial que en los ensayos precedentes no se había considerado: la orientación espacial.

Estudios llevados a cabo por Linn \& Petersen (1985) analizaban diferencias en las habilidades espaciales por sexo, motivo por el cual en nuestra prueba se presentan los resultados en función del género de los participantes, siguiendo el modelo de las experiencias en cursos similares (Contero, et.al., 2005; Contero, et.al., 2006, Martín-Dorta 2009, Martín 2010).

\section{Participantes}

En la prueba ha participado una población de cincuenta y dos estudiantes voluntarios correspondientes al primer curso de las titulaciones de Ingeniería Técnica Agrícola y Licenciatura en Geografía, con edades comprendidas entre los dieciocho y los veintidós años, distribuidos en una población de treinta y un hombres y veintiuna mujeres.

\section{Hardware y Software}

El trabajo se ha desarrollado sobre ordenadores Pentium IV a $2.80 \mathrm{GHz}$ y $512 \mathrm{Mb}$ de RAM, con sistema operativo Windows XP, en el aula de informática del Departamento de Expresión Gráfica en Arquitectura e Ingeniería para los estudiantes de ingeniería y en el aula de informática de la Facultad de Geografía para los estudiantes de Geografía, en la Universidad de La Laguna.

Al realizarse el estudio en la Universidad de La laguna, en la isla de Tenerife, el software empleado fue la aplicación Web del visor del geoportal de la Infraestructura de Datos Espaciales de Canarias, IDE Canarias, de acceso libre y gratuito. Nos ofrece, a través de su línea de comandos, visualizaciones en 2D y 3D en una o dos ventanas, medición de distancias y 
superficies, obtención de perfiles transversales y cálculo de rutas, mostrando la información georeferenciada en distintos formatos: Orto foto express, Orto foto express de alta resolución, mapa topográfico 1/1000, mapa topográfico 1/5000, mapa callejero y Modelo Digital de Sombras; todo ello conectado a una base de datos de información temática con la que el usuario puede desarrollar cartografía temática.

\section{Medición de las habilidades espaciales}

Cada participante realiza, antes y después del experimento, los tres test de medición de habilidades espaciales: el Mental Rotation Test, el Differencial Aptitude Test - Spatial Rotation Subset (DAT-SR5) y el Perspective Taking / Spatial Orientation Test y. De este modo podemos evaluar los resultados de la prueba piloto.

El MRT (Mental Rotation Test) está compuesto por 20 ejercicios, cada uno de los cuales consta de 5 figuras. (Figura 1). La primera, situada a la izquierda, es la que se toma como referencia, y el objetivo es localizar dentro de las otras cuatro, situadas a la derecha, las dos que corresponden a la de figura de referencia después de haber girado alrededor de uno o dos de sus ejes. Para la realización de este test el tiempo máximo es de 6 minutos. Se divide en dos períodos de 3 minutos: el alumno comienza, realiza las dos primeras páginas (10 primeras figuras) y no continua con el ejercicio (las siguientes 2 páginas, 20 figuras finales) hasta que terminan los 3 minutos. La puntuación del test consiste en aplicar 2 puntos por cada línea de figuras correctamente resueltas, 1 punto si solo se localiza una de las rotaciones por línea y no se marca ninguna otra figura, y 0 puntos si alguna de las marcas es incorrecta. La puntuación máxima será, por tanto, de 40 puntos.

Figura 1

EJEMPLO DEL MENTAL ROTATION TEST

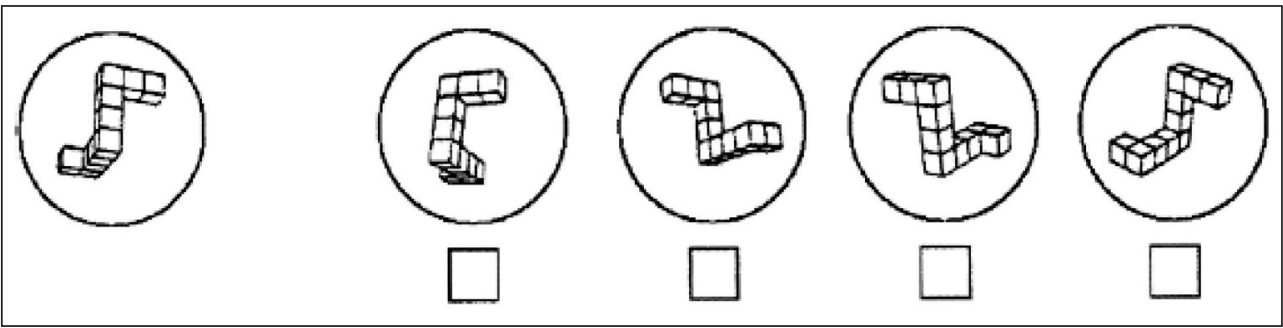

Fuente: CONTERO, M., et al. (2005): «Improving visualization skills in engineering education». IEE Computer Graphics and Applications. Vol. 25(5), 24-3.

El DAT-SR5 (Differencial Aptitude Test - Spatial Rotation Subset) consta de 50 ejercicios. En cada uno de ellos se presenta un modelo o patrón. A la derecha de cada modelo se ofrecen cuatro figuras de tres dimensiones. (Figura 2). El alumno debe averiguar cual de esas figuras es la única que ha podido formarse a partir del modelo. Éste siempre presenta la parte exterior de la figura. La puntuación de este test es de 1 punto por respuesta acertada. La puntuación máxima será de 50 puntos. 
Figura 2

EJEMPLO DEL DIFFERENCIAL APTITUDE TEST

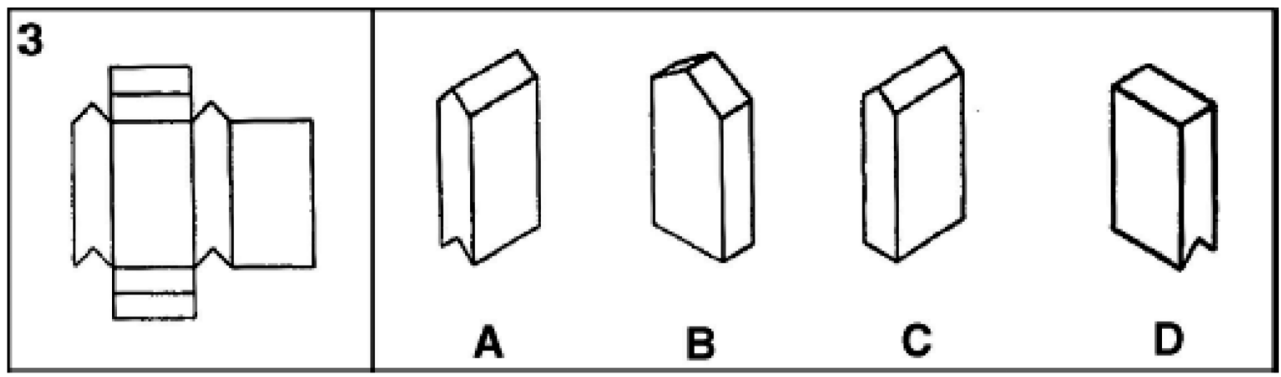

Fuente: CONTERO, M., et al. (2005): «Improving visualization skills in engineering education». IEE Computer Graphics and Applications. Vol. 25(5), 24-3.

Figura 3

EJEMPLO DEL SPATIAL ORIENTATION TEST

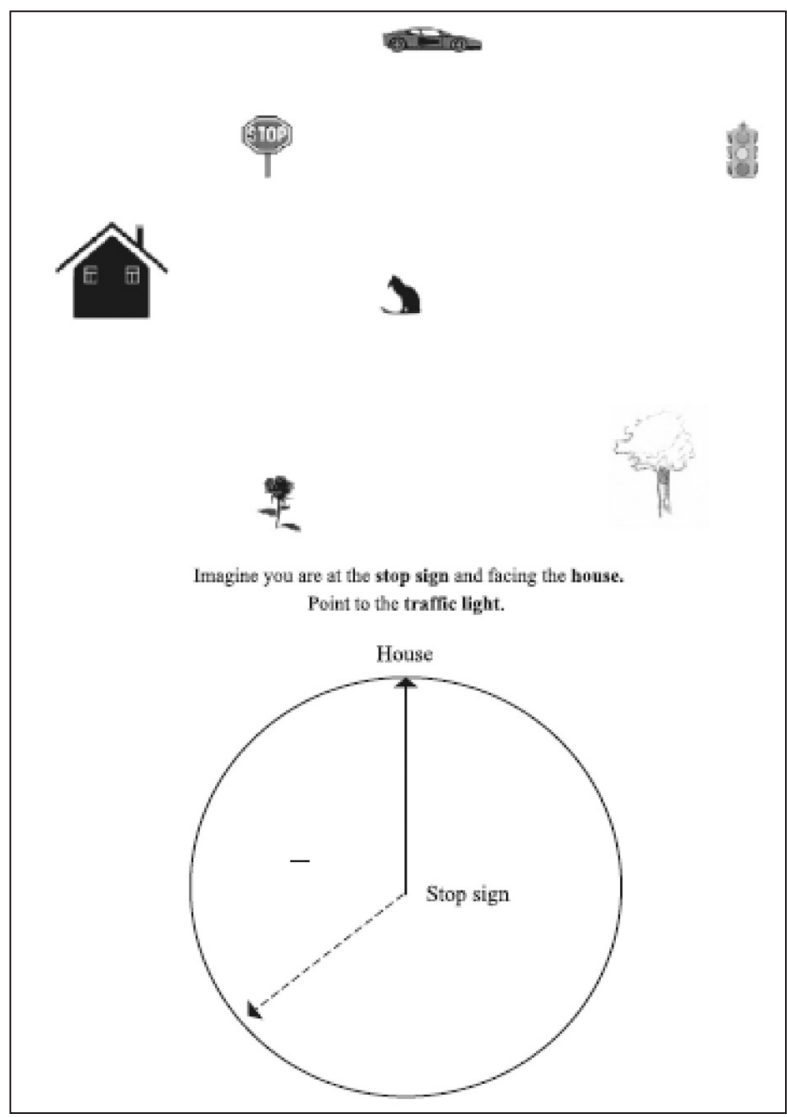

Fuente: University of California, Santa Bárbara (2008). 
El Perspective Taking/Spatial Orientation Test consiste en una serie de doce preguntas. En la parte superior se muestran un conjunto de siete objetos y en la parte inferior un círculo con una dirección indicada. (Figura 3). Para cada una de las 12 preguntas o ítems que se plantean, el usuario debe imaginar que está en un objeto del conjunto (que pasará a estar en el centro del círculo) y frente a otro objeto (que pasará a estar en la parte superior del círculo). El participante trazará una flecha desde el objeto del centro indicando la dirección a un tercer objeto desde la nueva orientación. La puntuación de cada ítem es la desviación absoluta en grados sexagesimales entre la respuesta del participante y la respuesta correcta (error direccional absoluto). La puntuación total será la media de las desviaciones de las 12 posiciones planteadas. Si el participante no responde a una pregunta, se le asigna un valor de $90^{\circ}$ para esta pregunta. Es importante destacar que este criterio establece, por tanto, que a menor puntuación mayor acierto. Para la resolución de este test es preciso que los participantes no giren en ningún momento el papel para evitar proporcionarle otra perspectiva. Se dispone de cinco minutos para su resolución.

\section{Instrucción}

El curso está dividido en dos fases de 5 y 3 horas respectivamente (Tabla 3).

Tabla 3

PLANIFICACIÓN DEL CURSO

\begin{tabular}{|l|c|c|c|}
\hline \multicolumn{1}{|c|}{ Fase } & Nivel & Descripción & Tiempo \\
\hline \multirow{2}{*}{ 1. Iniciación } & 1 & Introducción & $3 \mathrm{~h}$ \\
\cline { 2 - 4 } & 2 & Entrenamiento & $2 \mathrm{~h}$ \\
\hline \multirow{2}{*}{ 2. Perfeccionamiento } & 1 & Revisión & $1 / 2 \mathrm{~h}$ \\
\cline { 2 - 4 } & 2 & Ejercicio práctico (5 bloques) & $2 \mathrm{1} / 2 \mathrm{~h}$ \\
\hline
\end{tabular}

a) Fase 1. Iniciación

Nivel 1. Introducción (3 horas): Descripción de la plataforma IDE Canarias y explicación de las aplicaciones. Los estudiantes aprenden el funcionamiento de los comandos y contenidos midiendo distancias, superficies, obteniendo perfiles y visualizando el terreno a través de distintas modalidades en dos y tres dimensiones, a la vez que hacen consultas a la base de datos.

En la consecución de esta primera fase podemos destacar:

- En muchos casos se trata de estudiantes que tienen su primer contacto con cartografía e imágenes del territorio en formato digital georefenciadas e interconectadas a una base de datos temática: es la primera vez que utilizan este formato de Sistema de Información Geográfica (SIG).

- A pesar de la anterior afirmación, la mayoría de los estudiantes no parecen tener dificultad en el uso de la aplicación. Un 77\% de los alumnos estaban de acuerdo cuando se les preguntaba si se han sentido capaces de resolver los ejercicios planteados, dato recogido en la encuesta de satisfacción. 
Nivel 2. Entrenamiento (2 horas): Este nivel se diseña al objeto de que el alumno practique en casa con las herramientas que ha manejado en clase. Se le propone analizar la evolución geográfica de una zona a elegir por el estudiante, quien entrega al profesor un documento consistente en un máximo de diez hojas entre cartografía e imágenes en el que deberán quedar reflejados los cambios que la zona analizada ha ido experimentando a lo largo del tiempo. De este modo, el participante se familiariza con las distintas opciones de visualización en dos formatos: cartográfico e imágenes, ejercitando su capacidad de orientación espacial en ambos formatos.

b) Fase 2. Perfeccionamiento

Nivel 1. Revisión de trabajos realizados (1/2 hora): Se revisan y comentan los trabajos desarrollados en el nivel dos, proyectándolos en pantallas con cañón de vídeo.

Nivel 2: Ejercicio práctico. $(2 \mathrm{~h} 30 \mathrm{~m})$ : Se propone un ejercicio en el que se plantean cinco bloques de preguntas, cada uno de ellos pensado para que el alumno maneje todos los comandos y realice consultas a la base de datos de la IDE Canarias:

- Bloque I: Medición. En este bloque al alumno se le preguntan cuestiones en las que ha de operar con los comandos de medición de distancias, superficies y desniveles.

- Bloque II: Orientación: Aquí se le plantean situaciones en las que el alumno debe ejercitar sus dotes de orientación espacial para resolver el problema planteado. Se le pide, por ejemplo, indicar dónde estaba el sol en el momento de la toma de un lugar determinado utilizando ortofotos para su visualización, realizar recorridos con pequeño denominador de escala en el que tiene que ir reconociendo lugares que conoce empleando para el recorrido de ida el formato cartográfico y para el modo de vuelta ortofotos, o realizar el mismo recorrido desactivando y activando la posición del Norte Geográfico.

- Bloque III. Consulta. Consistente en un bloque de preguntas en las que el alumno debe acceder a los contenidos de la base de datos georeferenciada. Se le preguntan datos en torno a variables contempladas en la base de datos de la IDE Canarias.

- Bloque IV. Escenario posicional: en este apartado se le plantea elegir entre distintos formatos $2 \mathrm{~d}$ y $3 \mathrm{~d}$ en torno a una localización estática, es decir, un lugar determinado.

- Bloque V. Escenario dinámico: al igual que en el caso anterior, se plantea al alumno elegir entre distintos formatos de visualización $2 \mathrm{~d}$ y $3 \mathrm{~d}$ pero sobre un determinado recorrido que ha de seguir.

\section{ANÁLISIS DE DATOS Y RESULTADOS}

\section{Habilidades espaciales}

Antes y después de la realización del curso se evaluaron las capacidades espaciales de los estudiantes. En la tabla 4 se muestran las puntuaciones obtenidas y las ganancias medias para los test MRT, Perspective Taking/Spatial Orientation Test y DAT-SR5. 
La calificación del Perspective Taking/Spatial Orientation Test es la desviación en grados sexagesimales respecto de la respuesta correcta, razón por la cual a menor puntuación mayor acierto.

Tabla 4

PUNTUACIONES OBTENIDAS Y GANANCIAS MEDIAS

\begin{tabular}{|c|c|c|c|c|c|c|c|c|c|}
\hline \multirow{2}{*}{ TEST } & \multicolumn{3}{|c|}{ Mental Rotation Test } & \multicolumn{2}{c|}{ Persp. Tak/Spat. Orient. Test } & \multicolumn{3}{c|}{ Differencial Aptitude Test } \\
\cline { 2 - 10 } & Pre-curso & Post-curso & Ganancia & Pre-curso & Post-curso & Ganancia & Pre-curso & Post-curso & Ganancia \\
\cline { 2 - 10 } & (s.d.) & (s.d.) & (s.d.) & (s.d.) & (s.d.) & (s.d.) & (s.d.) & (s.d.) & (s.d.) \\
\hline Total & 13,1 & 17,46 & 4,37 & 50,36 & 35,29 & 15,06 & 24,88 & 32,96 & 8,08 \\
n=52 & $(5,83)$ & $(7,37)$ & $(5,73)$ & $(30,22)$ & $(28,27)$ & $(20,02)$ & $(8,09)$ & $(9,78)$ & $(6,81)$ \\
\hline Hombre & 13,45 & 17,87 & 4,42 & 51,29 & 33,73 & 17,56 & 23,65 & 31,58 & 7,94 \\
n=31 & $(6,42)$ & $(8,04)$ & $(6,11)$ & $(33,72)$ & $(30,48)$ & $(21,02)$ & $(7,85)$ & $(10,28)$ & $(7,09)$ \\
\hline Mujer & 12,57 & 16,86 & 4,29 & 48,99 & 37,60 & 11,38 & 26,71 & 35,00 & 8,29 \\
n=21 & $(4,95)$ & $(6,4)$ & $(5,25)$ & $(24,89)$ & $(25,21)$ & $(18,33)$ & $(8,28)$ & $(8,84)$ & $(6,53)$ \\
\hline
\end{tabular}

s.d.: desviación estándar.

Las ganancias medias resultaron, por tanto, de un 4.37 para el Mental Rotation Test (MRT), de un 15.06 para el Perspective Taking/Spatial Orientation Test y de un 8.08 para el Differencial Aptitude Test (DAT-SR5). Al realizarse la prueba con alumnos de primer curso al comienzo del mismo y, por tanto, no haberse ejercitado con asignaturas de contenidos afines a los contemplados en la prueba, no se han encontrado diferencias significativas entre las dos titulaciones presentes en la población estudiada.

Para el análisis estadístico se parte de la hipótesis nula (H0): «los valores medios de habilidades espaciales no han variado después del curso». Se aplica la prueba t-Student para series emparejadas (resultados de los test antes y después del curso) y se obtienen los $p$-valores que representan la probabilidad de que dicha afirmación sea cierta ( $p$-valor MRT $=0,00000124087, p$-valor Persp. Taking/Spat. Orient. Test $=0,00000160455, p$-valor DAT-SR5=0,00000000001).

El nivel de significación no llega en ningún caso al 1\%o, por lo que se rechaza la hipótesis nula en todos los casos y podemos afirmar con un nivel de significación superior al 99.9\% que la variación media del grupo estudiado ha experimentado un aumento.

Existe, por tanto, un efecto del curso con la Infraestructura de Datos Espaciales de Canarias sobre el valor medio de las habilidades espaciales medidas por los test de Rotación Espacial (MRT), Orientación Espacial (Perspective Taking/Spatial Orientation Test) y Visión espacial (DAT-SR5); dicho efecto es el de aumentar las habilidades de los sujetos sometidos a entrenamiento.

Se observa, además, que las ganancias obtenidas en los test tienen un aumento similar por género, excepto en el caso del Perspective Taking/Spatial Orientation Test, donde el aumento es significativamente mayor en hombres que en mujeres (Tabla 5). Numerosos estudios plantean la diferencia de género en la orientación espacial (Coluccia y Louse, 2004). 
Tabla 5

GANANCIAS EN RESULTADOS DE TEST DE HABILIDADES ESPACIALES POR GÉNERO

\begin{tabular}{|c|c|c|}
\hline Ganancias por género & Hombre & Mujer \\
\hline Mental Rotation Test (MRT) & 4,42 & 4,29 \\
\hline $\begin{array}{c}\text { Spatial Orientation Test (Perspective } \\
\text { Taking/Spatial Orientation Test) }\end{array}$ & 17,56 & 11,38 \\
\hline Differencial Aptitude Test (DAT-SR5) & 7,94 & 8,29 \\
\hline
\end{tabular}

1. Comparación con resultados obtenidos en cursos de mejora de habilidades espaciales anteriores

En la Universidad de La Laguna, el grupo de investigación Dehaes (http://www.degarin.com/dehaes/) ha desarrollado diversos cursos de mejora de habilidades espaciales, empleando ejercicios de modelado 3D partiendo de vistas isométricas en papel, aplicacio-

Tabla 6

COMPARACIÓN CON EXPERIENCIAS PREVIAS EN VARIABLES DE RELACIÓN ESPACIAL Y VISIÓN ESPACIAL

\begin{tabular}{|c|c|c|c|c|c|c|c|}
\hline \multirow{3}{*}{ CURSO } & \multicolumn{3}{|c|}{ Mental Rotation Test } & \multicolumn{3}{|c|}{ Differential Aptitude Test } & \multirow{3}{*}{ Descripción } \\
\hline & Pre & Post & Ganancia & Pre & Post & Ganancia & \\
\hline & (s.d.) & (s.d.) & (s.d.) & (s.d.) & (s.d.) & (s.d.) & \\
\hline $\begin{array}{l}\text { Ejercicios en papel } \\
\qquad n=17\end{array}$ & $\begin{array}{c}8,18 \\
(4,60)\end{array}$ & $\begin{array}{l}13,53 \\
(6,12)\end{array}$ & $\begin{array}{c}5,35 \\
(4,35)\end{array}$ & $\begin{array}{l}28,47 \\
(8,57)\end{array}$ & $\begin{array}{l}39,35 \\
(10,09)\end{array}$ & $\begin{array}{l}10,88 \\
(5,48)\end{array}$ & $\begin{array}{l}\text { Modelado 3D partiendo de vistas } \\
\text { isométricas en papel }\end{array}$ \\
\hline $\begin{array}{l}\text { Ejercicios Web } \\
\quad n=15\end{array}$ & $\begin{array}{c}9,60 \\
(4,46)\end{array}$ & $\begin{array}{l}13,27 \\
(4,80)\end{array}$ & $\begin{array}{c}3,36 \\
(5,85)\end{array}$ & $\begin{array}{l}30,53 \\
(5,40)\end{array}$ & $\begin{array}{l}36,67 \\
(5,60)\end{array}$ & $\begin{array}{c}5,13 \\
(5,25)\end{array}$ & $\begin{array}{c}\text { Aplicaciones Web de reconstrucción } \\
\text { de piezas a partir de vistas }\end{array}$ \\
\hline $\begin{array}{l}\text { Aplicación E-cigro } \\
\qquad n=20\end{array}$ & $\begin{array}{c}7,85 \\
(3,56)\end{array}$ & $\begin{array}{l}12,05 \\
(5,33)\end{array}$ & $\begin{array}{c}4,20 \\
(4,49)\end{array}$ & $\begin{array}{l}33,00 \\
(6,26)\end{array}$ & $\begin{array}{l}40,40 \\
(8,92)\end{array}$ & $\begin{array}{c}7,40 \\
(5,92)\end{array}$ & $\begin{array}{c}\text { Aplicación de boceado por ordenador } \\
\text { desarrollado por el grupo de } \\
\text { investigación REGEO }\end{array}$ \\
\hline $\begin{array}{l}\text { Software Sketchup } \\
n=12\end{array}$ & $\begin{array}{l}10,58 \\
(4,32)\end{array}$ & $\begin{array}{l}16,83 \\
(7,77)\end{array}$ & $\begin{array}{c}6,25 \\
(6,55)\end{array}$ & $\begin{array}{l}31,00 \\
(7,41)\end{array}$ & $\begin{array}{l}43,42 \\
(6,24)\end{array}$ & $\begin{array}{l}12,42 \\
(7,59)\end{array}$ & $\begin{array}{l}\text { Software de modelado 3D desarrollado } \\
\text { por Google }\end{array}$ \\
\hline $\begin{array}{c}\text { Videojuegos en PC } \\
n=21\end{array}$ & $\begin{array}{l}20,76 \\
(8,01)\end{array}$ & $\begin{array}{l}27,48 \\
(9,03)\end{array}$ & $\begin{array}{c}6,72 \\
(4,54)\end{array}$ & $\begin{array}{c}40,38 \\
(11,51)\end{array}$ & $\begin{array}{l}49,67 \\
(8,68)\end{array}$ & $\begin{array}{c}9,29 \\
(5,09)\end{array}$ & $\begin{array}{c}\text { Videojuegos donde se ejercita la } \\
\text { capacidad espacial: Tetris, Tetris } \\
\text { Revolution y Block 3D }\end{array}$ \\
\hline $\begin{array}{c}\text { Nintendo DS } \\
n=14\end{array}$ & $\begin{array}{l}12,43 \\
(5,73)\end{array}$ & $\begin{array}{l}22,79 \\
(9,19)\end{array}$ & $\begin{array}{l}10,36 \\
(4,76)\end{array}$ & $\begin{array}{l}43,14 \\
(8,30)\end{array}$ & $\begin{array}{l}52,07 \\
(8,40)\end{array}$ & $\begin{array}{c}8,93 \\
(4,76)\end{array}$ & $\begin{array}{c}\text { Videojuegos donde se ejercita la } \\
\text { capacidad espacial: Tetris, Táctil-Torre } \\
\text { y Puzzle }\end{array}$ \\
\hline $\begin{array}{l}\text { Realidad Aumentada } \\
\qquad n=25\end{array}$ & $\begin{array}{l}19,67 \\
(7,91)\end{array}$ & $\begin{array}{l}27,71 \\
(7,83)\end{array}$ & $\begin{array}{c}8,04 \\
(5,31)\end{array}$ & $\begin{array}{l}29,17 \\
(7,29)\end{array}$ & $\begin{array}{l}38,46 \\
(7,05)\end{array}$ & $\begin{array}{c}9,29 \\
(4,08)\end{array}$ & $\begin{array}{l}\text { Vistas 3D de piezas a través de } \\
\text { Realidad Aumentada }\end{array}$ \\
\hline $\begin{array}{l}\text { IDE } \\
n=52\end{array}$ & $\begin{array}{l}12,57 \\
(4,95)\end{array}$ & $\begin{array}{c}16,86 \\
(6,4)\end{array}$ & $\begin{array}{c}4,29 \\
(5,25)\end{array}$ & $\begin{array}{l}24,88 \\
(8,09)\end{array}$ & $\begin{array}{l}32,96 \\
(9,78)\end{array}$ & $\begin{array}{c}8,08 \\
(6,81)\end{array}$ & Desarrollado en este artículo \\
\hline
\end{tabular}

s.d.: desviación estándar. 
nes Web para la mejora de la visión espacial, una aplicación de bocetado por ordenador desarrollada por el grupo de investigación Regeo (http://www.regeo.uji.es/) denominada e-CIGRO, Software Google Sketchup, video juegos y, finalmente, una herramienta basada en la tecnología de realidad aumentada desarrollada conjuntamente por el grupo de investigación DEHAES y el equipo Human Centered Techonology de la Universidad Politécnica de Valencia.

En estos cursos se han analizado dos componentes de la capacidad espacial, las relaciones espaciales y la visión espacial, medidas con los test MRT y DAT-SR5 respectivamente, sin considerar la componente de la orientación espacial, por lo que la comparación de este curso con experiencias previas solo la podemos hacer en torno a estas dos variables (Tabla 6).

Respecto a la tercera componente, la orientación espacial, no se disponen de datos de experiencias previas en cursos de mejora. Sí existen resultados del Test de Orientación Espacial recogidos en una experiencia con 71 estudiantes de la Universidad de California (Kozhevnikov \& Hegarty, 2001), a los que se les midió la capacidad de orientación espacial con el test empleado en esta experiencia, el Perspective Taking/Spatial Orientation Test, obteniendo unos valores medios de 33,2 puntos con una desviación estándar de 23,3 (Tabla 7).

Tabla 7

COMPARACIÓN CON RESULTADOS PREVIOS EN EL PERSPECTIVE TAKING/SPATIAL ORIENTATION TEST

\begin{tabular}{|c|c|c|}
\hline \multicolumn{3}{|c|}{ Perspective Taking/Spatial Orientation Test } \\
\hline \multicolumn{2}{|c|}{ Universidad de La Laguna, Tenerife } & Universidad de Santa Bárbara, California \\
\hline Pre-curso & Post-curso & Experiencia Kozhelnikov-Heragrty \\
(s.d.) & (s.d.) & $($ s.d.) \\
50,36 & 35,29 & 33,20 \\
$(30,22)$ & $(28,27)$ & $(23,30)$ \\
\hline \multicolumn{2}{|c|}{$\mathrm{n}=52$} & $\mathrm{n}=71$ \\
\hline
\end{tabular}

s.d.: desviación estándar.

Fuente. Resultados Universidad de Santa Bárbara, California: Kozhevnikov and Hegarty (2001).

\section{Análisis de satisfacción del usuario}

La norma ISO/IEC 9241-11 define Satisfacción como «La libertad del usuario para mostrar su conformidad/disconformidad y sus actitudes hacia la utilización de un producto».

Los resultados de la satisfacción del usuario se recogen de la encuesta diseñada a tal efecto, y que se le facilita al alumno en formato papel. Las preguntas se han creado utilizando una escala tipo Likert, según la cual, a cada pregunta el encuestado le asignará una valoración numérica, valor que indica el grado de acuerdo o desacuerdo con respecto a la pregunta en una escala de cinco puntos. De esta forma se responde al cuestionario valorando con precisión el grado de acuerdo sobre las afirmaciones (Brooke, 1996). 
Escala de Likert:

\begin{tabular}{cl} 
Valor & Equivalencia \\
\hline 1 & Totalmente en desacuerdo \\
2 & En desacuerdo \\
3 & Ni de acuerdo ni en desacuerdo \\
4 & De acuerdo \\
5 & Totalmente de acuerdo
\end{tabular}

Las cuestiones que se plantearon en la encuesta fueron:

1 El curso ofrece un contenido útil

2 El curso me ha ayudado a comprender mejor el concepto de escala de un plano

3 El curso me ha ayudado a comprender mejor el espacio en planta y alzado

4 Ahora comprendo mejor la representación del relieve tanto en 2D como en 3D

5 La Herramienta de visualización en 3D me ayuda a interpretar lo representado en un mapa

6 Ahora comprendo mejor el concepto de coordenadas Geográficas Longitud y Latitud

7 Ahora comprendo mejor el concepto de coordenada U.T.M.

8 Creo que el curso ha mejorado mi capacidad de orientación espacial

9 Creo que el curso ha mejorado mis habilidades espaciales

10 Creo que es una potente herramienta para mi desarrollo como estudiante

11 ¿Recomendarías este curso a otros compañeros?

12 En términos generales: estoy satisfecho con el curso

La figura 4 muestra los resultados de los valores medios de cada pregunta.

Figura 4

RESULTADOS ENCUESTA DE SATISFACCIÓN EN LA ESCALA DE LIKERT

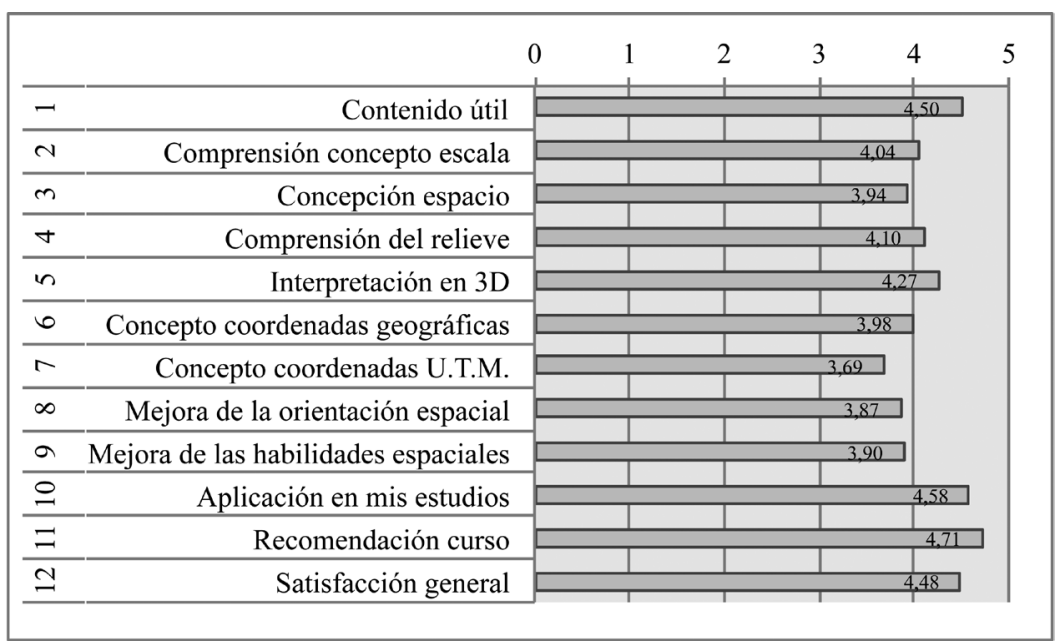


Las opiniones vertidas en la encuesta arrojan un alto grado de satisfacción del usuario respecto del curso. El alumno opina que el curso le ha ayudado a asimilar conceptos tales como la escala y los diferentes sistemas de coordenadas empleados en cartografía. Percibe, a su vez, que ha contribuido a mejorar sus habilidades espaciales y a interpretar mejor el espacio y el relieve empleando los distintos formatos de visualización que ofrece la Infraestructura de Datos Espaciales. Considera, además, que la herramienta puede resultar de gran interés para su desarrollo como estudiante.

Las puntuaciones obtenidas sobre el grado de satisfacción general (4.48) y sobre la recomendación del curso a otros compañeros (4.71) son indicadoras de la medida de la satisfacción general del usuario.

Bevan (2006) menciona que para hacer estimaciones fiables de los resultados de satisfacción son necesarios entre ocho y diez participantes, aunque muestras más grandes ofrecen un valor más significativo de los resultados, como es el caso que nos ocupa, en el que han participado cincuenta y dos estudiantes.

\section{CONCLUSIONES Y FUTUROS TRABAJOS}

Una vez finalizado el curso de mejora de habilidades espaciales mediante el uso de infraestructuras de datos espaciales, y a la vista de los resultados obtenidos en los test, fichas de datos y encuestas de satisfacción, podemos concluir:

- Las habilidades espaciales se pueden mejorar mediante entrenamiento específico. A la luz de los resultados obtenidos y el análisis realizado, el conjunto de actividades desarrolladas en el curso de mejora con el geoportal de la Infraestructura de Datos Espaciales de Canarias ha resultado ser una buena opción.

- Este modelo de curso de mejora puede suponer un aumento de los niveles de capacidad espacial. La ganancia media fue de 4,37 puntos en la componente de rotación espacial y de 8,08 puntos en la componente de visión espacial.

- La capacidad de orientación espacial se ve significativamente mejorada tras la consecución del curso, con un aumento medio de 15,06 puntos.

- El aumento en la capacidad espacial se produce de forma similar en mujeres y hombres para las variables de rotación espacial y de visión espacial. En cambio, el aumento de la componente de orientación espacial manifiesta ser mayor en hombres que en mujeres, con un aumento de 11,38 puntos en mujeres y de 17,56 en hombres.

- La satisfacción del alumnado con respecto al curso ha sido muy buena, lo cual invita a preparar estrategias de innovación docente en torno a nuevas tecnologías de información geográfica (TIG).

La información geográfica está cambiando de formato. En el contexto de esta investigación se plantean analizar otros servicios de difusión de información geográfica como pueden ser la aplicación Web Google Earth y el software Google SketchUp. Dada su sencillez de uso y accesibilidad consideramos que podría ser una buena herramienta para la docencia universitaria.

En el terreno del hardware, sería interesante estudiar cómo interactúa el usuario con modelización tridimensional del terreno bajo realidad aumentada, así como analizar las posi- 
bilidades que ofrecen los dispositivos de pantalla táctil de nueva generación como por ejemplo los Ipad, en la consulta, visualización y actualización de información geográfica.

\section{BIBLIOGRAFÍA}

BEVAN, N. (2006): «Practical Issues in usability measurement». Interactions, $\mathrm{n}^{\circ}$ 13(6), 42-43.

BROOKE, J. SUS, A. (1996): «A quick and dirty usability scale. User information Architecture A/D Group», en Digital Equipment Co, London, 22 Hand Court. 1996. URL: <http:// www.usability.serco.com/trump/documents/Suschapt.doc $>$. [Nov. 2009]

BENNET, G., SEASHORE, H., \& WESMAN, A. (2000). DAT 5: Test de aptitudes diferenciales. Madrid. TEA Ediciones.

BURNET, S., LANE, D. (1980): «Effects of academic instruction on spatial visualization». Intelligence, $\mathrm{n}^{\mathrm{O}} 4,233-242$.

CLEMENTS, D., BATISTA, M. (1992): «Geometry and Spatial Reasoning» en Handbook of Research on Mathematics Teaching and Learning (A.E. Kelly, \& R.A. Lesh). New York. Macmillian Publishing Company. 420-464.

CONTERO, M., COMPANY, P., SAORIN, NAYA, F. (2006): «Learning supports tools for developing spatial abilities in engineering design». International Journal of Engineering Education. vol. 22 (3), 470-477.

CONTERO, M., NAYA, F., COMPANY, P., SAORIN, J., CONESA, J. (2005): «Improving visualization skills in engineering education». IEEE Computer Graphics and Applications. vol. 25 (5), 24-3.

CORTIZO, T. (2007): «Protocolo para la normalización del tratamiento geográfico de la Información (TGGI)». Boletín de la A.G.E., $\mathrm{n}^{\circ}$ 45, 279-305.

DEVON, R., ENGLE, R., FOSTER, R. SATHIANATHAN, D. TURNER, G. (1994): «The effect of solid modelling software on 3D visualization skills». Engineering Design Graphics Journal, no $58,4-11$.

DURÁN, D. (2005): Educación geográfica. Cambios y continuidades. Buenos Aires. Lugar Editorial.

FERGUSON, E.E. (1992): Engineering and the Mind's Eye. MIT Press.

GARCÍA, J.M. (2008): «Las citas internacionales de las revistas españolas de geografía». Boletín de la A.G.E. no 46, 207-225.

GERSON, H., SHERYL, S., WISOCKI, Q. BAARTMANS,B. (2001): «The Development and Assessment of Multimedia Software for Improving 3-D Spatial Visualization Skills». Computer Applications in Engineering Education», $\mathrm{n}^{\circ} 9$ (2), 105-113.

HEGARTY, M.,KOHEVNIKOV, M., WALLER, D. (2008): «Perspective Taking/Spatial Orientation Test». University of California, Santa Barbara.

HEGARTY, M., WALLER, D. (2003): «A dissociation Between Mental Rotation and Perspective-Taking Spatial Abilities». Inteligence, $\mathrm{n}^{\circ}$ 32(2), 175-191.

HERRERO, C. (2001): «La docencia, uno de los oficios del Geógrafo». Boletín de la AG.E., $\mathrm{n}^{\circ} 31,185.191$.

KOZHEVNIKOV, M., HEGARTY, M. (2001): «A dissociation Between Object Manipulation, Spacial Ability and Spatial Orientation Ability». Memory and Cognition, $\mathrm{n}^{\circ}$ 29(5), 745-756. 
LINN, M., PETERSEN, A. (1985): «Emergence and Characterization of Sex Differences in Spatial Ability: A Meta-Analysis». Child Development, $\mathrm{n}^{\circ}$ 56, 1479-1498.

MAFALDA, R., (2000): Efeitos do uso de diferentes métodos de representaçao gráfica no desenvolvimento da habilidade de visualizaçao espacial. San Paolo, Portugal. Universidade de Sao Paulo.

MAIER, P. (1998): «Spatial Geometry and Spatial Ability: How to Make Solid Geometry Solid?» en Selected papers from the Annual Conference of Didactics of Mathematics. Osnabrück, E. Cohors-Fresenborg, K. Reiss, G. Toener, \& H. Weigand Eds., 63-75.

MARTÍN-DORTA, N. (2009) Análisis del uso de dispositivos móviles en el desarrollo de estrategias de mejora de las habilidades espaciales. Tesis doctoral dirigida por Manuel Contero González y José Luís Saorín Pérez. Valencia. Universidad Politécnica de Valencia. 253 p.

MARTÍN-DORTA, N., SAORÍN, J., CONTERO, M. (2008): «Development of a Fast Remedial Course to Improve the Spatial Abilities of Engineering Students». Journal of Engineering Education , n ${ }^{\circ} 97$ (4), 505-513.

MARTÍN, J. (2010): Estudio y evaluación de contenidos didácticos en el desarrollo de las habilidades espaciales en el ámbito de la Ingeniería. Tesis doctoral dirigida por Manuel Contero González y José Luís Saorín Pérez. Valencia. Universidad Politécnica de Valencia. 2010. 367 p.

McGEE, M.G. (1979): «Human Spatial Abilities: Psychometric Studies and Environmental, Genetic, Hormonal and Neurological Influences». Psychological Bulletin, nº 86 (5), 889918.

MEJÍA ÁVILA, D. (2008): «Sistemas de información geográfica, infraestructuras de datos espaciales y educación» en Mapping, $\mathrm{n}^{\circ}$ 125. URL: http://www.mappinginteractivo.com/ plantilla-ante.asp?id_articulo=1483 [enero 2010].

MILLER, C. (1996): «A historical review of applied and theoretical spatial visualization in engineering graphics». Emgineering Design Graphics Journal, nº 60(3), 12-33.

MINISTERIO DE LA PRESIDENCIA. Real Decreto 55/2005 de 21 de enero. Boletín Oficial del Estado. Número 21/2005. URL: http://www.crue.org/export/sites/Crue/legislacion/documentos/Estructura_ensenanzas/2._Real_Decreto_55-2005.pdf [marzo 2010]

MINISTERIO DE LA PRESIDENCIA. Real Decreto 1393/2007 de 29 de octubre. Boletín Oficial del Estado. Número 260. URL: http://www.boe.es/boe/dias/2007/10/30/pdfs/ A44037-44048.pdf [marzo 2010]

OLKUN, S. (2003): «Making connections: Improving spatial habilitéis with engineering drawing activities». International Journal for Mathematics Teaching and Learning. April, 1-10. URL: http://www.cimt.plymouth.ac.uk/journal/default.htm

PELLEGRINO, J., ALDERTON, D., SHUTE, V. (1984): «Understanding Spatial Ability». Educational Psychologist , nº 19 (3), 239-253.

REBER, A.S. (1985): Dictionary of Psychology. London. Penguin Boows.

RODRÍGUEZ, F. (2003): «Los documentos cartográficos como ejercicios-tipo para el control del conocimiento geográfico escolar». Boletín de la A.G.E., n 32, 263-271.

SÁNCHEZ, H., REYES, C. (2003): Psicología del aprendizaje y la educación superior. Lima, Perú. Ed. Visión Universitaria. 
SAORÍN, J.L. (2006): Estudio del efecto de la aplicación de tecnologías multimedia y del modelado basado en bocetos en el desarrollo de las habilidades espaciales. Tesis doctoral dirigida por Manuel Contero González. Valencia. Universidad Politécnica de Valencia. $243 \mathrm{p}$.

SMITH, I. (1964): Spatial ability: Its aducational and spatial significance. London. The University os London Press.

SORBY, S., BARARTMANS, J. (2000): «The Development and Assessment of a course for enhancing the 3D Spatial Visualization Kills of First Year Engineering Students». Journal of Engineering Education, vol. 89-3, 301-307.

SORBY, S., WYSOCKI, A., BAARTMANTS, B. (2003): Introduction to 3D Spatial Visualization: an active approach. Clifton Park, NY. Thomson Delmar Learning.

TORRES, Ma .C. (2003): «La geografía de la sociedad de la información: ¿real o virtual?». Boletín de la A.G.E., $\mathrm{n}^{\circ} 35,153-171$.

VANDERBERG, S., KUSE, A. (1978): «Mental Rotation, a Group test of three dimensional spatial visualization». Perceptual and motor Skills, no 47, 599-604.

VILLANUEVA, J. (2003): «La Geografía en la educación secundaria para adultos» Boletín de la A.G.E., n 35, 249-262. 
